\author{
ANNALES \\ POLONICI MATHEMATICI
}

$92.1(2007)$

\title{
A proof of the valuation property and preparation theorem
}

\author{
by KRzyszTof JAN NowaK (Kraków)
}

\begin{abstract}
The purpose of this article is to present a short model-theoretic proof of the valuation property for a polynomially bounded o-minimal theory $T$. The valuation property was conjectured by van den Dries, and proved for the polynomially bounded case by van den Dries-Speissegger and for the power bounded case by Tyne. Our proof uses the transfer principle for the theory $T_{\text {conv }}$ (i.e. $T$ with an extra unary symbol denoting a proper convex subring), which-together with quantifier elimination-is due to van den Dries-Lewenberg. The main tools applied here are saturation, the Marker-Steinhorn theorem on parameter reduction and heir-coheir amalgams.

The significance of the valuation property lies to a great extent in its geometric content: it is equivalent to the preparation theorem which says, roughly speaking, that every definable function of several variables depends piecewise on any fixed variable in a certain simple fashion. The latter originates in the work of Parusiński for subanalytic functions, and of Lion-Rolin for logarithmic-exponential functions. Van den Dries-Speissegger have proved the preparation theorem in the o-minimal setting (for functions definable in a polynomially bounded structure or logarithmic-exponential over such a structure). Also, the valuation property makes it possible to establish quantifier elimination for polynomially bounded expansions of the real field $\mathbb{R}$ with exponential function and logarithm.
\end{abstract}

1. Preliminaries. Throughout this article we deal with a polynomially bounded o-minimal theory $T$ in a first-order language $\mathcal{L}$ with field of exponents $K$ (which is a subfield of the field $\mathbb{R}$ of reals). The word "definable" in a structure $\mathcal{R}$ always means "definable with parameters from $\mathcal{R}$ "; "definable with no parameters" is called "0-definable". It is well known that one can always extend $\mathcal{L}$ and $T$ by definitions to:

$$
\begin{aligned}
\mathcal{L}^{\mathrm{df}}:= & \mathcal{L} \text { augmented by a new function symbol } f_{\varphi}(\bar{x}) \text { for each } \mathcal{L} \text {-formula } \\
& \varphi(\bar{x}, y) \text { such that } T \vdash \forall \bar{x} \exists y \varphi(\bar{x}, y), \\
T^{\mathrm{df}}:= & T \text { extended by the new defining axioms } \varphi\left(\bar{x}, f_{\varphi}(\bar{x})\right) .
\end{aligned}
$$

2000 Mathematics Subject Classification: 03C64, 14P15, 32B20.

Key words and phrases: o-minimal structures, valuation property, preparation theorem.

Research partially supported by KBM grant 1P03A 00527 and the RAAG Network. 
Every model $\mathcal{R}$ of $T$ expands uniquely to a model $\mathcal{R}^{\mathrm{df}}$ of $T^{\mathrm{df}}$. Since the theory $T$ has definable cell decomposition, $T^{\mathrm{df}}$ has quantifier elimination, and since $T$ has definable Skolem functions (which follows from cell decomposition as well), $T^{\mathrm{df}}$ has universal axiomatization. Consequently, $T$ has a prime model $\mathcal{P}$ which has a unique elementary embedding into every model $\mathcal{R}$ of $T$; the image of $\mathcal{P}$ in $\mathcal{R}$ consists of the interpretations of all constant symbols of the language $\mathcal{L}^{\mathrm{df}}$.

If $\mathcal{R}$ is an elementary substructure of a model $\mathcal{S}$ of $T: \mathcal{R} \prec \mathcal{S}$, then $\mathcal{R}^{\mathrm{df}}$ is a substructure of $\mathcal{S}^{\mathrm{df}}$ in the language $\mathcal{L}^{\mathrm{df}}: \mathcal{R}^{\mathrm{df}} \subset \mathcal{S}^{\mathrm{df}}$. For a subset $A \subset \mathcal{S}$, $\mathcal{R}\langle A\rangle$ denotes the definable closure of $A$ over $\mathcal{R}$ in $\mathcal{S}$, i.e. the substructure of $\mathcal{S}^{\text {df }}$ generated by $\mathcal{R} \cup A$ in the language $\mathcal{L}^{\mathrm{df}}$; of course $\mathcal{R}\langle A\rangle$ is an elementary substructure of $\mathcal{S}$. The operation of definable closure satisfies the ordinary axioms for the span operation (in particular the Steinitz exchange property), whence one can define $\operatorname{rank}, \operatorname{rk} \mathcal{R}$, or relative $\operatorname{rank}, \operatorname{rk}(\mathcal{S} / \mathcal{R})$, in an ordinary fashion.

Consider two ordered fields $\mathcal{R} \subset \mathcal{S}$. We say that $\mathcal{R}$ is Dedekind complete or tame in $\mathcal{S}$ if one of the three equivalent conditions is satisfied:

(i) the trace on $\mathcal{R}$ of every interval in $\mathcal{S}$ is an interval in $\mathcal{R}$;

(ii) the cut made in $\mathcal{R}$ by every element $s \in \mathcal{S}$ is rational;

(iii) for each $\mathcal{R}$-bounded element $s \in \mathcal{S}$, there is a unique element $r \in \mathcal{R}$ such that $s-r$ is an $\mathcal{R}$-infinitesimal; we call $r:=\operatorname{st}(s)$ the standard part of the element $s$.

Marker-Steinhorn Theorem on Parameter Reduction. Consider o-minimal structures $\mathcal{R} \prec \mathcal{S}$ with $\mathcal{R}$ Dedekind complete in $\mathcal{S}$. Then the trace $X \cap \mathcal{R}^{n}$ of every definable set $X \subset \mathcal{S}^{n}$ in $\mathcal{S}^{n}$ is definable in $\mathcal{R}^{n}$. In other words, the trace $X \cap \mathcal{R}^{n}$ can be defined by means of parameters from $\mathcal{R}$ only.

In this section we shall make use of the following well-known

Corollary (cf. [8, 1]). Under the previous assumptions, if $f: \mathcal{S}^{n} \rightarrow \mathcal{S}$ is a definable function, then the sets

$$
E_{-\infty}:=\left\{\bar{x} \in \mathcal{R}^{n}: f(\bar{x})<\mathcal{R}\right\}, \quad E_{+\infty}:=\left\{\bar{x} \in \mathcal{R}^{n}: f(\bar{x})>\mathcal{R}\right\}
$$

are definable, and the function

$$
\operatorname{st}(f(\bar{x})): \mathcal{R}^{n} \backslash\left(E_{-\infty} \cup E_{+\infty}\right) \rightarrow \mathcal{R}
$$

is definable.

Consider now the theory $T_{\text {conv }}$ of pairs $(\mathcal{R}, V)$, where $V$ is a convex subring of a model $\mathcal{R}$ of $T$, in the language $\mathcal{L}$ with an extra unary relation symbol to denote $V$ (cf. [2]). Every maximal elementary substructure $\mathcal{R}^{\prime}$ of $\mathcal{R}$ contained in $V$ is Dedekind complete and cofinal in $\mathcal{R}$, and isomorphic to the residue field $\bar{V}$ of $V$; furthermore, $V$ is the convex hull of $\mathcal{R}^{\prime}$ in $\mathcal{R}$. Van 
den Dries-Lewenberg [2] proved a relative version of quantifier elimination for $T_{\text {conv }}$ :

If $T$ has quantifier elimination and universal axiomatization, then $T_{\text {conv }}$ has quantifier elimination.

Hence $T_{\text {conv }}$ is a complete theory and we have at our disposal the transfer principle: in order to prove a theorem expressible in the first-order language $\mathcal{L}_{\text {conv }}$ for all models of $T_{\text {conv }}$, it suffices to prove it for one particular model.

The convex subring $V$ is a valuation ring in $\mathcal{R}$ with maximal ideal $\mathfrak{m}=\mathfrak{m}_{V}$ and valuation group $\Gamma=\Gamma_{V}$; let $v$ denote the induced valuation of the field $\mathcal{R}$. We now give a simple proof for the following proposition on stabilization of valuation due to van den Dries [1]. Our proof makes use of the transfer principle and piecewise uniform asymptotics only.

Proposition 1 (on stabilization of valuation). If $f: \mathcal{R} \rightarrow \mathcal{R}$ is a definable function, then there exists $u \in V$ such that for all $x \in V, x \geq u$, we have

$$
v(f(x))=v(f(u)) \quad \text { or equivalently } \quad \frac{f(x)}{f(u)} \in V \backslash \mathfrak{m} .
$$

Proof. The function $f$ is of the form

$$
f(x)=g\left(x, r_{1}, \ldots, r_{m}\right)
$$

where $g$ is a 0 -definable function and $\bar{r} \in \mathcal{R}^{m}$ are parameters. So we shall prove the statement for all parameters $\bar{r}$ from the model under consideration, $g$ being fixed. Since the assertion is expressible in the first-order language of the theory $T_{\text {conv }}$, we may assume that $\mathcal{R}=\mathcal{P}\langle a\rangle$, where $|\mathcal{P}|<a$ and $V=\widehat{\mathcal{P}}$ is the convex hull of $\mathcal{P}$ in $\mathcal{R}$. Then every parameter $r_{i}$ equals $h_{i}(a)$ for a 0-definable function $h_{i}: \mathcal{P} \rightarrow \mathcal{P}$. Putting

$$
k(x, y):=g\left(x, h_{1}(y), \ldots, h_{m}(y)\right),
$$

we are thus reduced to considering 0-definable functions $f(x)=k(x, a)$, $k: \mathcal{P} \times \mathcal{P} \rightarrow \mathcal{P}$.

In this case, in view of piecewise uniform asymptotics for polynomially bounded o-minimal theories, there exist an exponent $\mu \in K$, a $u \in \mathcal{P}$ and a 0 -definable function $c(x)$ such that for every $x \in \mathcal{P}, x \geq u$, we have

$$
\frac{k(x, y)}{c(x) y^{\mu}} \rightarrow 1 \quad \text { as } y \rightarrow \infty, y \in \mathcal{P}
$$

Hence for every $x, \varepsilon \in \mathcal{P}, x \geq u, \varepsilon>0$, we have

$$
\left|\frac{k(x, y)}{c(x) y^{\mu}}-1\right|<\varepsilon \quad \text { when } y \in \mathcal{P}, y \gg 0 \text {. }
$$


It follows by overspill that

$$
\left|\frac{k(x, a)}{c(x) a^{\mu}}-1\right|<\varepsilon \quad \text { for every } x \in V, x \geq u \text {. }
$$

Consequently, $v(f(x))=v(k(x, a))=\mu v(a)$ for all $x \in V, x \geq u$, as desired.

Let $f, g: \mathcal{R} \rightarrow \mathcal{R}$ be definable functions, and $\pi: V \rightarrow \bar{V}$ the canonical mapping onto the residue field $\bar{V}$ of $V$. We say that $f$ and $g$ are asymptotic on $V, f \underset{V}{\sim} g$, if either $f(x)=g(x)=0$ for $x \in V, x \gg 0$, or

$$
\frac{f(x)}{g(x)} \in V \quad \text { for } x \in V, x \gg 0, \quad \text { and } \quad \pi\left(\frac{f(x)}{g(x)}\right) \rightarrow 1 \quad \text { as } x \rightarrow \infty, x \in V \text {. }
$$

Corollary. There exist $\lambda \in K$ and $c \in \mathcal{R}$ such that $f(x) \underset{V}{\sim} c x^{\lambda}$. Consequently, for any $\varepsilon=1 / v$ with $v \in V, v>0$, there exist $u, w \in \mathcal{R}$, $u \in V, w>V$, such that $\left|f(x) /\left(c x^{\lambda}\right)-1\right|<\varepsilon$ for all $x \in[u, w]$.

Proof. Indeed, the maximal elementary substructure $\mathcal{R}^{\prime} \simeq \bar{V}$ of $\mathcal{R}$ contained in $V$ is Dedekind complete in $\mathcal{R}$. Taking $u \in \mathcal{R}^{\prime}$ as in Proposition 1, it follows from the Corollary to the Marker-Steinhorn theorem that the function

$$
\operatorname{st}\left(\frac{f(x)}{f(u)}\right): \mathcal{R}^{\prime} \rightarrow \mathcal{R}^{\prime}
$$

is $\mathcal{R}^{\prime}$-definable. Then it is asymptotic to $c^{\prime} x^{\lambda}$ for some $\lambda \in K$ and $c^{\prime} \in \mathcal{R}^{\prime}$ :

$$
\operatorname{st}\left(\frac{f(x)}{f(u) c^{\prime} x^{\lambda}}\right) \rightarrow 1 \quad \text { as } x \rightarrow \infty, x \in \mathcal{R}^{\prime} .
$$

Putting $c:=f(u) c^{\prime}$ completes the proof.

Consider now a model $(\mathcal{R}, V)$ of the theory $T_{\text {conv }}$ whose valuation $v$ has rank $d<\infty$. This means that the value group $\Gamma=\Gamma_{V}$ has $d+1$ isolated subgroups of the form

$$
\Gamma_{0}=(0) \subset \Gamma_{1} \subset \Gamma_{1} \oplus \Gamma_{2} \subset \cdots \subset \Gamma=\Gamma_{1} \oplus \Gamma_{2} \oplus \cdots \oplus \Gamma_{d},
$$

where the subgroups $\Gamma_{i}$ are archimedian such that $\Gamma_{1}^{+}<\Gamma_{2}^{+}<\cdots<\Gamma_{d}^{+}$. We have, of course, a one-to-one correspondence between these isolated subgroups and the prime ideals $\mathfrak{p}_{i}$ in $V$, as well as the convex subrings $V_{i}$ such that $V \subset V_{i} \subset \mathcal{R}$ :

$$
\begin{gathered}
\mathfrak{p}_{i}=\left\{x \in \mathcal{R}: v(x)>\Gamma_{i}\right\}, \quad V_{i}=\left\{x \in \mathcal{R}: v(x)>-\Gamma_{i+1}\right\}=V_{\mathfrak{p}_{i}}, \\
\mathfrak{p}_{0}=\mathfrak{m} \supset \mathfrak{p}_{1} \supset \cdots \supset \mathfrak{p}_{d-1} \supset \mathfrak{p}_{d}=(0), \quad V_{0}=V \subset V_{1} \subset \cdots \subset V_{d-1} \subset V_{d}=\mathcal{R} .
\end{gathered}
$$

The valuation group of $V_{i}$ is isomorphic to $\Gamma_{i+1} \oplus \cdots \oplus \Gamma_{d}$; the valuation group of $V_{d}=\mathcal{R}$ is $(0)$. 
Observation. There exists an elementary extension $\left(\mathcal{R}^{*}, V^{*}\right)$ of $(\mathcal{R}, V)$ the value group $\Gamma^{*}$ of which is of the form

$$
\Gamma^{*}=\underbrace{\mathbb{R} \oplus \cdots \oplus \mathbb{R}}_{d \text { times }} .
$$

Such an elementary extension can be obtained by a successive adjunction of elements from an $\aleph_{0}$-saturation of $(\mathcal{R}, V)$. Here we sketch that procedure.

Every archimedian group $\Gamma_{i}$ may be regarded as a subgroup of the additive group $\mathbb{R}$ of real numbers; we may assume that $1 \in \Gamma_{i}$. Take any number $\delta \in \mathbb{R} \backslash \Gamma_{i}$; then $\delta$ makes an irrational cut $C$ in $\Gamma_{i}$. One can lift $C$ to a unique cut $\widetilde{C}:=\{x \in \mathcal{R}: x \leq 0$ or $(x>0, v(x)>C)\}$ in $\mathcal{R}$, and next adjoin to $\mathcal{R}$ an element $a$ which realizes the cut $\widetilde{C}$. We get an elementary extension $(\mathcal{R}\langle a\rangle, W)$ of $(\mathcal{R}, V)$; clearly, $w(a)$ realizes the cut $C$ in $\Gamma_{i}$ as well. One must show that the valuation $w$ with value group $\Gamma_{W}$ obtained in this fashion is also of rank $d$. This is due to the control over definable functions in the vicinity of the cuts made by the convex subrings $V_{i}$ in $\mathcal{R}$, as described in the Corollary to Proposition 1. Indeed, every definable function $f: \mathcal{R} \rightarrow \mathcal{R}$ is asymptotic in each $V_{i}$ to a function $c x^{\lambda}$. Hence $\left|f(x) /\left(c x^{\lambda}\right)-1\right|<1 / 2$ for all $x$ from an interval $[u, w]$ with $u \in V_{i}, w>V_{i}$, and this inequality extends to the cuts made by the subrings $V_{i}$ in $\mathcal{R}$. Therefore, for every $\mathcal{R}$-definable function $g$, the element $b=g(a)$ can realize none of those cuts, and consequently $w(b)=w(g(a))$ can realize no cut in $\Gamma$ made by the isolated subgroups $\Gamma_{i}$. Otherwise the element $w(b)$ would generate yet another isolated subgroup of $\Gamma_{W}$. Further, if $a=f(b)$, we would get $\left|f(a) /\left(c a^{\lambda}\right)-1\right|<1 / 2$, and thus

$$
w(a)=v(c)+\lambda w(b) \in \Gamma+K w(b),
$$

whence $w(b) \in \Gamma+K w(a)$. But this is impossible since the group $\Gamma+K w(a)$, on a par with the group $\Gamma$, has exactly $d+1$ isolated subgroups whenever $w(a)$ defines an irrational cut in $\Gamma$.

Repeating successively the above procedure for each subgroup $\Gamma_{i}$ and all real numbers, we obtain an increasing chain of elementary extensions (of cardinality $\leq$ power of the continuum). By the Tarski-Vaught lemma, the union of this chain is the desired elementary extension $\left(\mathcal{R}^{*}, V^{*}\right)$ of $(\mathcal{R}, V)$.

Applying the transfer principle and saturated models, we shall prove the following

Proposition 2. If a definable function $f: \mathcal{R} \rightarrow \mathcal{R}$ is constant on no interval in $\mathcal{R}$, then there exist $s \in \mathcal{R}, \lambda \in K \backslash\{0\}$ and $c \in \mathcal{R}$ such that

$$
f(x)-s \underset{V}{\sim} c x^{\lambda} .
$$

Consequently, for any $\varepsilon=1 / v$ with $v \in V, v>0$, there exist $u, w \in \mathcal{R}$, $u \in V, w>V$, such that $\left|f(x)-s /\left(c x^{\lambda}\right)-1\right|<\varepsilon$ for all $x \in[u, w]$. 
Proof. In view of the Corollary to Proposition 1, our statement is equivalent to the following first-order sentence:

$$
\exists s \in \mathcal{R} \forall u \in V \exists x_{1}, x_{2} \in V, x_{1}, x_{2}>u \quad\left|\frac{f\left(x_{1}\right)-s}{f\left(x_{2}\right)-s}\right|>2 ;
$$

here the number 2 may be replaced by any real number $>1$. Via the transfer principle, it suffices to consider one model of the theory $T_{\text {conv }}$. Take a model $(\mathcal{R}, V)$ with $V=\widehat{\mathcal{P}}=$ convex hull of the prime model $\mathcal{P}$ in $\mathcal{R}$, where $\mathcal{R}$ is an $\alpha^{+}$-saturated model of the theory $T$ with $\alpha=$ cofinality of $\mathcal{P}$. For simplicity we confine ourselves to the case $\alpha=\aleph_{0}$; the general case goes the same way, but with transfinite induction instead of an ordinary induction argument.

We prove Proposition 2 for the above model by reductio ad absurdum. Suppose the contrary, i.e. for any $s \in \mathcal{R}$ the function $f(x)-s \underset{V}{\widetilde{C}} c$ is asymptotic to a constant function $c$, i.e. $\lambda=0$; obviously, $c \neq 0$.

We then assert that $f(x)-s \in c(1+\mathfrak{m})$ for all $x \in V, x \gg 0$. For otherwise, if $\mathcal{R}^{\prime} \simeq \bar{V}$ is a maximal elementary substructure of $\mathcal{R}$ contained in $V$, the function

$$
\mathrm{st}\left(\frac{f(x)-s}{c}\right): \mathcal{R}^{\prime} \rightarrow \mathcal{R}^{\prime}
$$

would be asymptotic to 1 but $\neq 1$ ultimately in $\mathcal{R}^{\prime}$. So $(f(x)-s) / c-1$ would be asymptotic to $d x^{\lambda}$ with $d \in \mathcal{R}^{\prime}, \lambda \in K, \lambda<0$, and consequently

$$
f(x)-s-c \underset{V}{\sim} c d x^{\lambda},
$$

contrary to our hypothesis. Therefore $\operatorname{st}((f(x)-s) / c)=1$ ultimately in $\mathcal{R}^{\prime}$, and thus $f(x)-s \in c(1+\mathfrak{m})$ for all $x \in V, x \gg 0$, as asserted.

Take any $a_{0} \in V$; then there is some $c_{0} \in \mathcal{R}$ such that

$$
f(x)-f\left(a_{0}\right) \underset{V}{\sim} c_{0}, \quad f(x)-f\left(a_{0}\right) \in c_{0}(1+\mathfrak{m})
$$

for all $x \in V, x \geq a_{1}$ with $a_{1} \in V$. The last condition is equivalent to $\left|\left(f(x)-f\left(a_{0}\right)-c_{0}\right) / c_{0}\right|<\varepsilon_{k}$ for all $k$, where the $1 / \varepsilon_{k}$ form a cofinal sequence in $V$. Each of these inequalities extends (by overspill) to an interval $\left[a_{1}, b_{1 k}\right]$ with $b_{1 k}>V$. Since $\mathcal{R}$ is $\alpha^{+}$-saturated, there is an element $b_{1} \in \mathcal{R}$ such that $1 / \varepsilon_{k}<b_{1}<b_{1 k}$ for all $k \in \mathbb{N}$. Clearly, $V<b_{1}$ and

$$
f(x)-f\left(a_{0}\right) \in c_{0}(1+\mathfrak{m}) \quad \text { for all } x \in\left[a_{1}, b_{1}\right] .
$$

Next we get $f(x)-f\left(a_{1}\right) \in c_{0} \cdot \mathfrak{m}$ for $x \in\left[a_{1}, b_{1}\right]$, whence, as before,

$$
f(x)-f\left(a_{1}\right) \underset{V}{\sim} c_{1} \in c_{0} \cdot \mathfrak{m} \quad \text { and } \quad f(x)-f\left(a_{1}\right) \in c_{1}(1+\mathfrak{m})
$$

for all $x \in\left[a_{2}, b_{2}\right]$ with $a_{2}, b_{2} \in \mathcal{R}, a_{2} \in V, b_{2}>V$.

By induction we can construct three sequences $\left(a_{n}\right),\left(b_{n}\right),\left(c_{n}\right)$ of elements of $\mathcal{R}$ such that $a_{n} \in V, a_{n}>1 / \varepsilon_{n}$ are cofinal in $V, b_{n}>V$, the sequence 
$\left(v\left(c_{n}\right)\right)$ is strictly increasing, and

$$
v\left(f(x)-f\left(a_{n}\right)\right)=v\left(c_{n}\right) \quad \text { for all } x \in\left[a_{n+1}, b_{n+1}\right] .
$$

Since $\mathcal{R}$ is $\alpha^{+}$-saturated, we can find an element $b \in \mathcal{R}$ such that $a_{n}<b<b_{n}$ for all $n \in \mathbb{N}$. Then $v\left(f(b)-f\left(a_{n}\right)\right)=v\left(c_{n}\right)$ is a strictly increasing sequence, and thus the valuation $v(g(x))$ of the function $g(x):=f(b)-f(x)$ does not stabilize. This contradiction with Proposition 1 completes the proof.

\section{Valuation group of a simple extension}

Proposition 3. Let $\mathcal{R}$ be a finitely generated and polynomially bounded structure, $V \subset \mathcal{R}$ a convex subring of $\mathcal{R}$, and $v: \mathcal{R} \backslash\{0\} \rightarrow \Gamma=\Gamma_{V}$ the induced valuation. Then

(i) $\operatorname{rk} v \leq \operatorname{dim}_{K} \Gamma \leq \operatorname{rk} \mathcal{R}$.

(ii) If $(\mathcal{R}, V) \subset(\mathcal{R}\langle a\rangle, W)$, then $\operatorname{dim}_{K} \Gamma_{W} \leq \operatorname{dim}_{K} \Gamma+1$; more precisely, $\Gamma_{W}=\Gamma \oplus K w(a)$ whenever $w(a) \notin \Gamma$.

Proof. We proceed by induction on $\mathrm{rk} \mathcal{R}$ and prove point (ii), which is the induction step. By induction hypothesis, rk $v=$ : $d \leq \mathrm{rk} \mathcal{R}<\infty$. Then $\Gamma=\Gamma_{V}=\Gamma_{1} \oplus \cdots \oplus \Gamma_{d}$, and we can find-as described in the observation of Section 1 -an elementary extension $\left(\mathcal{R}^{*}, V^{*}\right)$ of $(\mathcal{R}, V)$ such that

$$
\Gamma^{*}=\underbrace{\mathbb{R} \oplus \cdots \oplus \mathbb{R}}_{d \text { times }} .
$$

Consider now an heir-coheir amalgam of elementary extensions

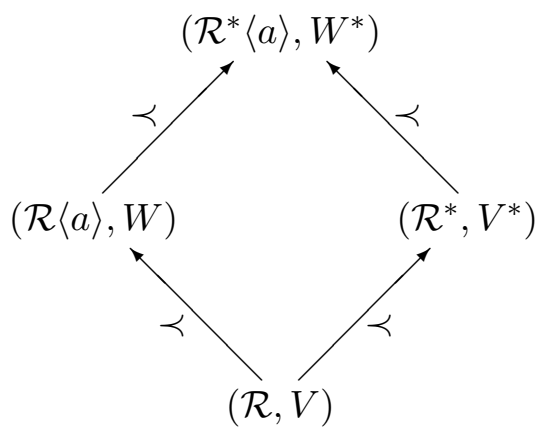

Clearly, $w^{*}(a) \notin \Gamma^{*}$ if $w(a) \notin \Gamma$. Indeed, were $w^{*}(a) \in \Gamma^{*}$, then

$$
\exists c^{*} \in \mathcal{R}^{*} \quad w^{*}(a)=w^{*}\left(c^{*}\right), \quad \text { i.e. } \quad \frac{a}{c^{*}} \in W^{*} \backslash \mathfrak{m}_{W^{*}},
$$

and thus, by the heir-coheir property,

$$
\exists c \in \mathcal{R} \quad w(a)=w(c), \quad \text { i.e. } \quad \frac{a}{c} \in W \backslash \mathfrak{m}_{W},
$$

which is a contradiction. 
It suffices to establish (ii) for the elementary extension

$$
\left(\mathcal{R}^{*}, V^{*}\right) \subset\left(\mathcal{R}^{*}\langle a\rangle, W^{*}\right) .
$$

Indeed, let $b=f(a)$, where $f: \mathcal{R} \rightarrow \mathcal{R}$ is a definable function. Suppose $w^{*}(b) \in \Gamma^{*}+\lambda w^{*}(a)$ for a $\lambda \in K$, whence

$$
\exists c^{*} \in \mathcal{R}^{*} \quad w^{*}(b)=w^{*}\left(c^{*} a^{\lambda}\right), \quad \text { i.e. } \quad \frac{f(a)}{c^{*} a^{\lambda}} \in W^{*} \backslash \mathfrak{m}_{W^{*}} .
$$

It follows from the heir-coheir property that

$$
\exists c \in \mathcal{R} \quad w(b)=w\left(c a^{\lambda}\right), \quad \text { i.e. } \quad \frac{f(a)}{c a^{\lambda}} \in W \backslash \mathfrak{m}_{W},
$$

and thus $w(b) \in \Gamma+\lambda w(a)$, as required.

Now we shall show that if $w^{*}(a) \notin \Gamma^{*}$, then for every $b \in \mathcal{R}^{*}\langle a\rangle$ we have

$$
w^{*}(b) \in \Gamma^{*}+K w^{*}(a) .
$$

Since $\Gamma^{*}$ is the direct sum of a finite number of copies of $\mathbb{R}$, and the ordered set $\mathbb{R}$ of real numbers is Dedekind complete, there exists an element $\gamma=$ $v^{*}(r) \in \Gamma^{*}, r \in \mathcal{R}^{*}$, such that $w^{*}(a)-w^{*}(r)$ realizes the cut made by an isolated subgroup of $\Gamma^{*}$ in $\Gamma^{*}$. For, if the cut made by $w^{*}(a)$ lies inside of a $\Gamma_{i}^{*} \simeq \mathbb{R}$, we take the real number $\delta \in \Gamma_{i}^{*}$ which is closest to $w^{*}(a)$. Hence $\left|w^{*}(a)-\delta\right|<\left(\Gamma_{i}^{*}\right)^{+}$, and either we are done or we repeat the reasoning for a $\Gamma_{j}^{*}$ with $j<i$.

Therefore the element $a / r$ realizes the cut made by a convex subring of $\mathcal{R}^{*}$ in $\mathcal{R}^{*}$. Clearly, $b=f(a / r)$ for some definable function $f: \mathcal{R}^{*} \rightarrow \mathcal{R}^{*}$. It follows from the Corollary to Proposition 1 that

$$
\begin{aligned}
w^{*}(b) & =w^{*}(f(a / r))=w^{*}\left(c(a / r)^{\lambda}\right) \\
& =w^{*}(c)-\lambda w^{*}(r)+\lambda w^{*}(a) \in \Gamma^{*}+K w^{*}(a),
\end{aligned}
$$

concluding the proof.

Corollary. Consider a polynomially bounded, o-minimal theory $T$ and a simple extension $(\mathcal{R}, V) \subset(\mathcal{R}\langle a\rangle, W)$ of models of the theory $T_{\text {conv }}$. Then $\operatorname{dim}_{K} \Gamma_{W} \leq \operatorname{dim}_{K} \Gamma_{V}+1$; more precisely, $\Gamma_{W}=\Gamma_{V} \oplus K w(a)$ whenever $w(a) \notin \Gamma_{V}$.

Proof. Indeed, if $b \in \mathcal{R}\langle a\rangle$, then $b \in \mathcal{R}^{\prime}\langle a\rangle$ for a finitely generated substructure $\mathcal{R}^{\prime} \prec \mathcal{R}, \operatorname{rk} \mathcal{R}^{\prime}<\infty$; let $V^{\prime}:=V \cap \mathcal{R}^{\prime}$ and $\Gamma_{V^{\prime}}$ be its valuation group. By Proposition 3, we get $w(b) \in \Gamma_{V^{\prime}}+K w(a) \subset \Gamma_{V}+K w(a)$, as asserted.

REMARK. Proposition 3 immediately implies a stronger inequality (cf. $[1$, Section 5]):

If $\operatorname{rk} \mathcal{R}<\infty$ and $\bar{V}$ is the residue field of the convex subring $V$, then $\operatorname{rk} \bar{V}+\operatorname{dim}_{K} \Gamma_{V} \leq \operatorname{rk} \mathcal{R}$. 
From the above one can derive the following Wilkie inequality (loc. cit.) through an argument of Wilkie (cf. [12]), based on saturated models and an iteration procedure:

Suppose $T$ is a polynomially bounded theory and $(\mathcal{R}, V) \prec(\mathcal{S}, W)$ are models of $T_{\text {conv }}$ with $\operatorname{rk}(\mathcal{S} / \mathcal{R}) \leq \infty$. Then

$$
\operatorname{dim}_{K}\left(\Gamma_{W} / \Gamma_{V}\right)+\operatorname{rk}(\bar{W} / \bar{V}) \leq \operatorname{rk}(\mathcal{S} / \mathcal{R}) .
$$

\section{Valuation property and preparation theorem}

VAluation Property. Consider a polynomially bounded, o-minimal theory $T$ and a simple extension

$$
(\mathcal{R}, V) \subset(\mathcal{R}\langle a\rangle, W)
$$

of models of the theory $T_{\text {conv }}$ with valuation groups $\Gamma_{V}$ and $\Gamma_{W}$, respectively. If $\Gamma_{V} \neq \Gamma_{W}$, then there is an $r \in \mathcal{R}$ for which $w(a-r) \notin \Gamma_{V}$.

Proof. We may assume that $\operatorname{rk} \mathcal{R}<\infty$, because $w(b) \notin \Gamma_{V}$ for some $b \in \mathcal{R}^{\prime}\langle a\rangle$, where $\mathcal{R}^{\prime}$ is a finitely generated substructure of $\mathcal{R}$. Now, if for some $r \in \mathcal{R}^{\prime}$ the valuation $w^{\prime}(a-r)$ does not belong to the valuation group $\Gamma_{V^{\prime}}$ of the restriction $v^{\prime}:=v \mid \mathcal{R}^{\prime}$, it follows from Proposition 3 that

$$
w^{\prime}(a-r)=\gamma+\lambda w^{\prime}(b)
$$

for some $\lambda \in K, \lambda \neq 0$, and $\gamma \in \Gamma_{V^{\prime}}$. Hence $w(a-r) \notin \Gamma_{V}$.

Consider, as in the proof of Proposition 3, an heir-coheir amalgam of elementary extensions

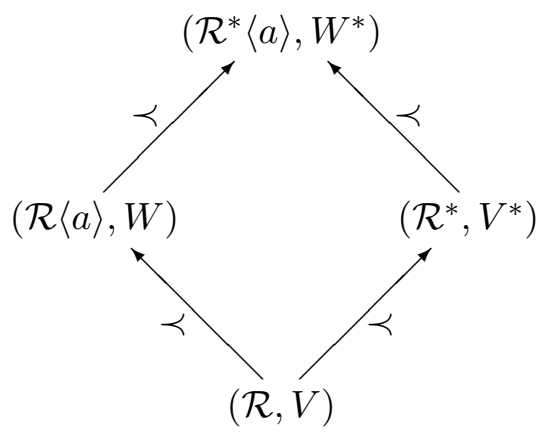

It suffices to establish the valuation property for the simple extension $\left(\mathcal{R}^{*}, V^{*}\right) \subset\left(\mathcal{R}^{*}\langle a\rangle, W^{*}\right)$. Indeed, $w^{*}(b) \notin \Gamma_{V^{*}}$, for otherwise

$$
\exists r^{*} \in \mathcal{R}^{*} \quad w^{*}(b)=v^{*}\left(r^{*}\right),
$$

and thus, by the heir-coheir property, we would get a contradiction

$$
\exists r \in \mathcal{R} \quad w(b)=v(r) \in \Gamma_{V} .
$$

Further, if $w^{*}\left(a-r^{*}\right) \notin \Gamma_{W^{*}}$, then $w^{*}\left(a-r^{*}\right) \in \Gamma_{V^{*}}+\lambda w^{*}(b)$ for some $\lambda \in K, \lambda \neq 0$, whence 


$$
\exists r^{*}, s^{*} \in \mathcal{R}^{*} \quad w^{*}\left(a-r^{*}\right)=v^{*}\left(s^{*}\right)+\lambda w^{*}(b) .
$$

Therefore, again by the heir-coheir property, we get the required result

$$
\exists r, s \in \mathcal{R} \quad w(a-r)=v(s)+\lambda w(b) \notin \Gamma_{V} .
$$

We thus have to show that if $w^{*}(b) \notin \Gamma_{V^{*}}$, then $w^{*}(a-r) \notin \Gamma_{V^{*}}$ for some $r \in \mathcal{R}^{*}$. Since the valuation group $\Gamma_{V^{*}}$ is the direct sum of a finite number of copies of $\mathbb{R}$ (which are Dedekind complete), we deduce - as in the proof of Proposition 3-that for some $v^{*}(r) \in \Gamma_{V^{*}}, r \in \mathcal{R}^{*}$, the element $w^{*}(b)-v^{*}(r)$ realizes the cut made by an isolated subgroup of $\Gamma_{V^{*}}$ in $\Gamma_{V^{*}}$. Replacing $b$ by $b / r$ we may, of course, assume that $b$ realizes the cut made by a convex subring of $\mathcal{R}^{*}$ in $\mathcal{R}^{*}$. Clearly, $a=f(b)$ for some definable function $f: \mathcal{R}^{*} \rightarrow \mathcal{R}^{*}$ which is constant on no interval in $\mathcal{R}^{*}$. Now it follows from Proposition 2 that there exist $s \in \mathcal{R}^{*}, \lambda \in K, \lambda \neq 0$, and $c \in \mathcal{R}^{*}$ such that

$$
w^{*}(a-r)=w^{*}(f(b)-r)=w^{*}\left(c b^{\lambda}\right)=w^{*}(c)+\lambda w^{*}(b) \notin \Gamma_{V^{*}}^{*},
$$

which completes the proof.

The valuation property yields, via a routine compactness argument, the preparation theorem for one variable:

Corollary. Let $\mathcal{R}$ be a polynomially bounded, o-minimal structure, $f: \mathcal{R} \rightarrow \mathcal{R}$ be a definable function and $\varepsilon \in \mathbb{Q}, \varepsilon>0$. Then there exist

$$
\lambda_{1}, \ldots, \lambda_{k} \in K, \quad r_{1}, \ldots, r_{k}, c_{1}, \ldots, c_{k} \in \mathcal{R}
$$

such that for all $x \in \mathcal{R}$ we have

$$
f(x)=\left|x-r_{i}\right|^{\lambda_{i}} \cdot c_{i} \cdot u
$$

for an $i=1, \ldots, k$ and some $u \in \mathcal{R}$ with $|u-1|<\varepsilon$.

Proof. Indeed, by passing to the theory $T^{\mathrm{df}}$ in the extended language $\mathcal{L}^{\text {df }}$, we shall deal with models $\mathcal{S}$ which are elementary extensions of $\mathcal{R}$, $\mathcal{R} \prec \mathcal{S}$. Through compactness, we must show that for each $a \in \mathcal{S}$ there exist $\lambda \in K, r, c \in \mathcal{R}$ and $u \in \mathcal{S},|u-1|<\varepsilon$, such that

$$
f(a)=|a-r|^{\lambda} \cdot c \cdot u \text {. }
$$

Consider now, as the convex subrings of $\mathcal{R}, \mathcal{R}\langle a\rangle$ and $\mathcal{S}$, respectively, the convex hulls of the field of real numbers $\mathbb{R}$ in these fields. Then it follows from the valuation property and the Corollary to Proposition 3 that there exist $\lambda \in K, r, c \in \mathcal{R}$ and $u \in \mathcal{S}$ such that

$$
w(u)=0, \quad f(a)=|a-r|^{\lambda} \cdot c \cdot u .
$$

Since $w(u)=0$, we see that $u$ is of the form $u_{0}+\mathbb{R}$-infinitesimal, and thus $\left|u / u_{0}-1\right|<\varepsilon$. Replacing $c$ and $u$ with $u_{0} c$ and $u / u_{0}$, respectively, we get the desired result. 
The general version below can immediately be obtained through modeltheoretic compactness and definable choice:

Preparation TheOREM. Under the previous assumptions, consider a definable function $f: \mathcal{R}^{n+1} \rightarrow \mathcal{R}$ and an $\varepsilon \in \mathbb{Q}, \varepsilon>0$. Then there exist $\lambda_{1}, \ldots, \lambda_{k} \in K$ and definable functions

$$
r_{1}, \ldots, r_{k}, c_{1}, \ldots, c_{k}: \mathcal{R}^{n} \rightarrow \mathcal{R}, \quad u_{1}, \ldots, u_{k}: \mathcal{R}^{n+1} \rightarrow(1-\varepsilon, 1+\varepsilon) \subset \mathcal{R}
$$

such that for all $\bar{t} \in \mathcal{R}^{n}$ and $x \in \mathcal{R}$ we have

$$
f(\bar{t}, x)=\left|x-r_{i}(\bar{t})\right|^{\lambda_{i}} \cdot c_{i}(\bar{t}) \cdot u_{i}(\bar{t}, x) \quad \text { for an } i=1, \ldots, k .
$$

\section{References}

[1] L. van den Dries, T-convexity and tame extensions II, J. Symbolic Logic 62 (1997), $14-34$.

[2] L. van den Dries and A. Lewenberg, T-convexity and tame extensions, ibid. 60 (1995), 74-102.

[3] L. van den Dries, A. Macintyre and D. Marker, The elementary theory of restricted analytic fields with exponentiation, Ann. of Math. 140 (1994), 183-205.

[4] L. van den Dries and P. Speissegger, The field of reals with multisummable series and the exponential function, Proc. London Math. Soc. (3) 81 (2000), 513-565.

[5] - - - o-minimal preparation theorems, in: Proc. Euro-Conference in Model Theory and its Applications, Ravello, 2002.

[6] W. Hodges, Model Theory, Cambridge Univ. Press, Cambridge, 1997.

[7] J.-M. Lion et J.-P. Rolin, Théorème de préparation pour les fonctions logarithmicoexponentielles, Ann. Inst. Fourier (Grenoble) 47 (1997), 859-884.

[8] D. Marker and C. Steinhorn, Definable types in o-minimal theories, J. Symbolic Logic 59 (1994), 185-198.

[9] A. Parusiński, Lipschitz stratification of subanalytic sets, Ann. Sci. École Norm. Sup. 27 (1994), 661-696.

[10] -, On the preparation theorem for subanalytic functions, in: New Developments in Singularity Theory (Cambridge, 2000), NATO Sci. Ser. II Math. Phys. Chem. 21, Kluwer, Dordrecht, 2001, 193-215.

[11] J. Tyne, T-levels and T-convexity, $\mathrm{PhD}$ thesis, Univ. of Illinois at Urbana-Champaign, 2003.

[12] A. J. Wilkie, Model completeness results for expansions of the ordered field of real numbers by restricted Pfaffian functions and the exponential function, J. Amer. Math. Soc. 9 (1996), 1051-1094.

Institute of Mathematics

Jagiellonian University

Reymonta 4

30-059 Kraków, Poland

E-mail: nowak@im.uj.edu.pl

Received 14.3.2007

and in final form 30.4.2007 